ARTICLE

\title{
Highly nonlinear trion-polaritons in a monolayer semiconductor
}

\author{
R. P. A. Emmanuele ${ }^{1}$, M. Sich (10) ${ }^{1}$, O. Kyriienko ${ }^{2,3 凶}$, V. Shahnazaryann ${ }^{3,4}$, F. Withers (1) ${ }^{5}$, A. Catanzaro ${ }^{1}$, \\ P. M. Walker ${ }^{1}$, F. A. Benimetskiy ${ }^{3}$, M. S. Skolnick ${ }^{1,3}$, A. I. Tartakovskii (i) ${ }^{1}$, I. A. Shelykh ${ }^{3,6}$ \& \\ D. N. Krizhanovskii (1, $1,3 \times$
}

Highly nonlinear optical materials with strong effective photon-photon interactions are required for ultrafast and quantum optical signal processing circuitry. Here we report strong Kerr-like nonlinearities by employing efficient optical transitions of charged excitons (trions) observed in semiconducting transition metal dichalcogenides (TMDCs). By hybridising trions in monolayer $\mathrm{MoSe}_{2}$ at low electron densities with a microcavity mode, we realise trionpolaritons exhibiting significant energy shifts at small photon fluxes due to phase space filling. We find the ratio of trion- to neutral exciton-polariton interaction strength is in the range from 10 to 100 in TMDC materials and that trion-polariton nonlinearity is comparable to that in other polariton systems. The results are in good agreement with a theory accounting for the composite nature of excitons and trions and deviation of their statistics from that of ideal bosons and fermions. Our findings open a way to scalable quantum optics applications with TMDCs.

\footnotetext{
${ }^{1}$ Department of Physics and Astronomy, The University of Sheffield, Sheffield S3 7RH, UK. ${ }^{2}$ Department of Physics and Astronomy, University of Exeter, Stocker Road, Exeter EX4 4QL, UK. ${ }^{3}$ Department of Physics and Engineering, ITMO University, St. Petersburg 197101, Russia. ${ }^{4}$ Institute of Physics, Polish Academy of Sciences, Al. Lotnikow 32/46, 02-668 Warsaw, Poland. ${ }^{5}$ College of Engineering, Mathematics and Physical Sciences, University of Exeter, Exeter EX4 4QF, UK. ${ }^{6}$ Science Institute, University of Iceland, Dunhagi-3, IS-107 Reykjavik, Iceland. ${ }^{\circledR}$ email: kyriienko@ukr.net; d.krizhanovskii@sheffield.ac.uk
} 
S trong optical Kerr nonlinearity can be realised in photonic systems where light is resonantly coupled with optically active medium ${ }^{1}$. These can correspond to optical transitions in semiconductor quantum dots ${ }^{2,3}$, single molecules ${ }^{4}$ and Rydberg atoms $s^{5}$; the scalability and integration into photonic devices, nevertheless, remains a significant challenge. These obstacles can be overcome by employing hybrid $2 \mathrm{D}$ exciton-photon $(2 \mathrm{D}$ polariton) systems 6 , , enabling large effective photon-photon interactions as well as scalability and ultrafast response ${ }^{8-11}$. So far nonlinear polaritons have been investigated in neutral exciton-polariton platform based on GaAs quantum wells and at low temperatures $(4-70 \mathrm{~K})$. Interaction-based effects such as polariton Bose-Einstein condensation and superfluidity ${ }^{6}$, solitons $^{12}$, quantum emission ${ }^{11,13,14}$ as well as polariton transistors/ switches ${ }^{15,16}$ have been reported.

Recently, layered materials such as graphene and transition metal dichalcogenides (TMDCs) ${ }^{17}$ have arisen as very promising optically active 2D media offering compatibility and ease of integration with various nanophotonic devices ${ }^{18}$. Optical bistability and regenerative oscillations have been demonstrated in hybrid Si-graphene microcavities ${ }^{19}$. In contrast to graphene, monolayers of TMDCs host Wannier-Mott excitons with a huge binding energy of about $200-500 \mathrm{meV}$ and large oscillator strength $^{20}$. This enables polariton formation in photonic structures with just a single TMDC monolayer ${ }^{21}$ and at room temperature 22,23 , offering a major advantage over other semiconductor polariton platforms (such as GaAs). Importantly, the strong Coulomb interactions give rise to very robust $2 \mathrm{D}$ trions (charged excitons) in TMDCs. The large oscillator strength of trions enables formation of well-resolved trion-polariton resonances $^{24}$ at relatively small electron density ${ }^{21,25}$, which, as we show here, leads to a pronounced phase space filling effect enabling nonlinearity of one to two orders of magnitude bigger (depending on exciton-photon detuning) than that of neutral exciton-polaritons in TMDC platform. Furthermore, the nonlinear refractive index $\left(n_{2}\right)$ per single TMDC monolayer due to trion-polaritons is estimated to be three to five orders of magnitude greater than in bare 2D TMDC materials and graphene studied in the weak light-matter coupling regime. We also probe nonlinearities due to neutral exciton-polaritons, which are observed to decrease by more than an order of magnitude with power. Such a result is explained by three-exciton and possibly trion-mediated exciton-exciton scattering processes. Overall, our work opens a new highly nonlinear system for quantum optics applications enabling in principle scalability and control through nano-engineering of van der Waals heterostructures.

\section{Results}

Experimental setup. In our experiment, a monolayer of $\mathrm{MoSe}_{2}$ (Fig. 1a) was placed into an open-access microcavity (MC) (see Fig. $1 c^{26}$ ) where Laguerre-Gauss (LG) photonic modes strongly couple to exciton and trion states forming polaritons. Figure $1 \mathrm{~b}$ shows the spectrum of PL emission from the monolayer. The strong peak at lower energy $\sim 1.62 \mathrm{eV}$ arises from the trion (T) emission due to the natural doping in the sample, while the weaker peak at $\sim 1.65 \mathrm{eV}$ corresponds to neutral (X) excitons. To characterise the polaritons the MC was excited with a laser at $1.95 \mathrm{eV}$ and polariton emission was recorded as a function of the detuning between the excitons and $L G_{00}$ mode. In Fig. $1 \mathrm{~d}$ the lower, middle and upper polariton branches (labelled LPB, MPB and UPB) are observed due to the strong coupling of the photon mode with $\mathrm{T}$ and $\mathrm{X}$, respectively. The peak polariton positions (black dots in Fig. 1d, e) were fitted using a model of three coupled oscillators (see Supplementary Information, Notes 1 and 3 , for the details).
Nonlinear trion-polaritons. In order to probe the polariton nonlinearity polaritons were excited resonantly using a laser with 100 -fs pulse duration. The excited polaritons then decay via photon emission through the DBRs with a rate $\gamma_{\mathrm{C}}|C|^{2}$ given by the cavity mode linewidth $\gamma_{\mathrm{C}} \sim 0.4 \mathrm{meV}\left(|C|^{2}\right.$ is the photonic fraction) or are absorbed in the $2 \mathrm{D}$ material with a rate given by the polariton linewidth $\gamma_{\text {pol }}\left(\gamma_{\text {pol }} \sim 3-5 \mathrm{meV} \gg \gamma_{\mathrm{C}}|C|^{2}\right)$ due to scattering with exciton disorder resulting in creation of an exciton-trion reservoir (Fig. 1f $)^{26,27}$. Measurements of the intensity of transmitted light enable us to estimate the polariton density excited inside the MC and the density of the reservoir. Monitoring the frequency shift of the polariton resonance with increasing pump power, we observe the influence of interaction between polaritons and the exciton-trion reservoir (Fig. 1f), and can extract the strength of exciton- and trion-based nonlinearity.

We first present the results for interacting trion-polaritons. In this experiment the laser energy is fixed at approximately the trion level, $E_{\mathrm{p}}=1.62 \mathrm{eV}$, and the transmission spectrum is recorded as the energy of the photon $L G_{00}$ mode is scanned through the trion resonance. Since the linewidth of the pulsed laser $(\sim 15 \mathrm{meV})$ is significantly larger than the observed photon-trion Rabi-splitting of $\hbar \Omega_{\mathrm{T}} \approx 5.8 \mathrm{meV}$, it was possible to inject a similar number of polaritons in the vicinity of the trion resonance for each cavity mode position. Excitation of the UPB is negligible since it is located at energies more than $\sim 30 \mathrm{meV}$ above the trion-polariton states.

Figure 2 shows the results of three cavity scans performed for different pump powers. At the lowest pulse power, $10 \mathrm{nW}$ (Fig. 2a), an anti-crossing between the cavity mode and the trion level is clearly observed, as in the case under the non-resonant excitation. With an increase of the pump power the photon-trion Rabi-splitting is reduced (Fig. 2b) leading to the blueshift and redshift of the LPB and MPB states in the vicinity of trion resonance (Fig. 2a, inset), respectively. At $P=70 \mathrm{nW}$ no anticrossing is observed (Fig. 2c), with MPB and LPB merging together and forming a single polariton branch. To quantify the strength of the nonlinearity, we recorded the spectra of the transmitted light as a function of pump power at a fixed photon mode energy near the trion resonance (Fig. 3a), at a fixed photon-exciton detuning $\delta_{\mathrm{C}-\mathrm{X}}=-15.4 \mathrm{meV}$. In Fig. 3a two peaks are observed at low power of $5 \mathrm{nW}$; the MPB peak has a higher intensity due to the higher photonic fraction. With increase of the pulse power, the MPB peak exhibits a fast redshift. The intensity of the trionlike LPB quickly reduces down to zero with power due to reduction of its photon fraction as the strong trion-photon coupling collapses.

The collapse of the strong coupling is driven by the population of both polaritons inside the cavity and reservoir determining the total density of excited excitons and trions $n_{\text {tot }}$ (see Supplementary Note 1.2, for estimation of $n_{\text {tot }}$ ). The reservoir may consist of localised or dark exciton/trion states as well as high-momenta trions degenerate with trion-polariton resonances 26,27 . For the case of resonantly driven trion-polaritons $n_{\text {tot }}$ is dominated mostly by the trion density $n_{\mathrm{T}}\left(n_{\text {tot }} \approx n_{\mathrm{T}}\right)$, since the peak of the exciton density of states is blue detuned by $\sim 30 \mathrm{meV}$ from the trion.

Figure $3 \mathrm{~b}$ shows that the MPB energy shifts rapidly to the red wavelengths with $n_{\text {tot }}$ at densities $n_{\text {tot }}<2 \times 10^{2} \mu \mathrm{m}^{-2}$ and then exhibits a plateau corresponding to the quenching of trion-photon coupling, followed by a gradual increase. From the redshift of the MPB branch in Fig. $3 \mathrm{~b}$ we plot (see Supplementary Note 1.4) the value of the Rabi-splitting $\hbar \Omega_{\mathrm{T}}$ vs $n_{\text {tot }}$ in Fig. $3 c$. The collapse of trion-photon coupling occurs at a small density of $n_{\text {tot }} \sim 2 \times 10^{2} \mu \mathrm{m}^{-2}$ very close to half the estimated density of free electrons $n_{\mathrm{e}} / 2 \approx 2 \times 10^{2} \mu \mathrm{m}^{-2}$ (see 

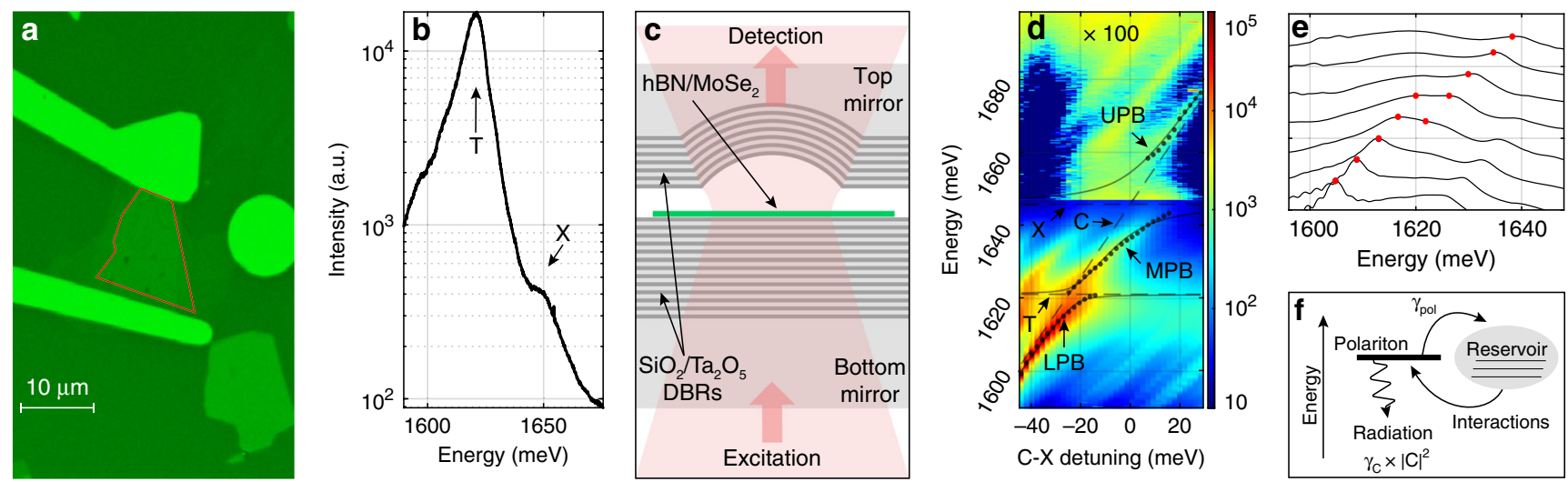

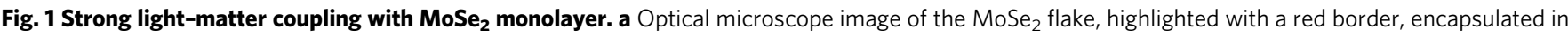
hBN and clamped with two golden contacts on top of the bottom mirror. $\mathbf{b}$ PL emission of the flake under off-resonant excitation with exciton ( $\mathrm{X}$ ) and trion (T) peaks highlighted. The observed order of magnitude difference for the intensity of the PL of the trion relative to the exciton arises from fast neutral exciton to trion states relaxation processes due to scattering with phonons and electrons. c Scheme of experiment for resonant excitation of the open cavity in the transmission geometry. $\mathbf{d}$ PL emission as a function of the detuning between bare cavity $L G_{00}$ mode (C) and exciton (X) plotted in logarithmic colour scale; black points (dots) are experimental peak positions of the polariton resonances extracted from the fitting of experimental spectra at each detuning with a Gaussian; solid lines are theoretical polariton branches. Higher order transverse LG modes are also observed on the left and right-hand side of $L G_{00}$ polariton states. e Normalised PL spectra around trion resonance, showing anticrossing behaviour. $\mathbf{f}$ Schematic showing emission and absorption of driven polariton resonance and interactions with the reservoir of trions and excitons.
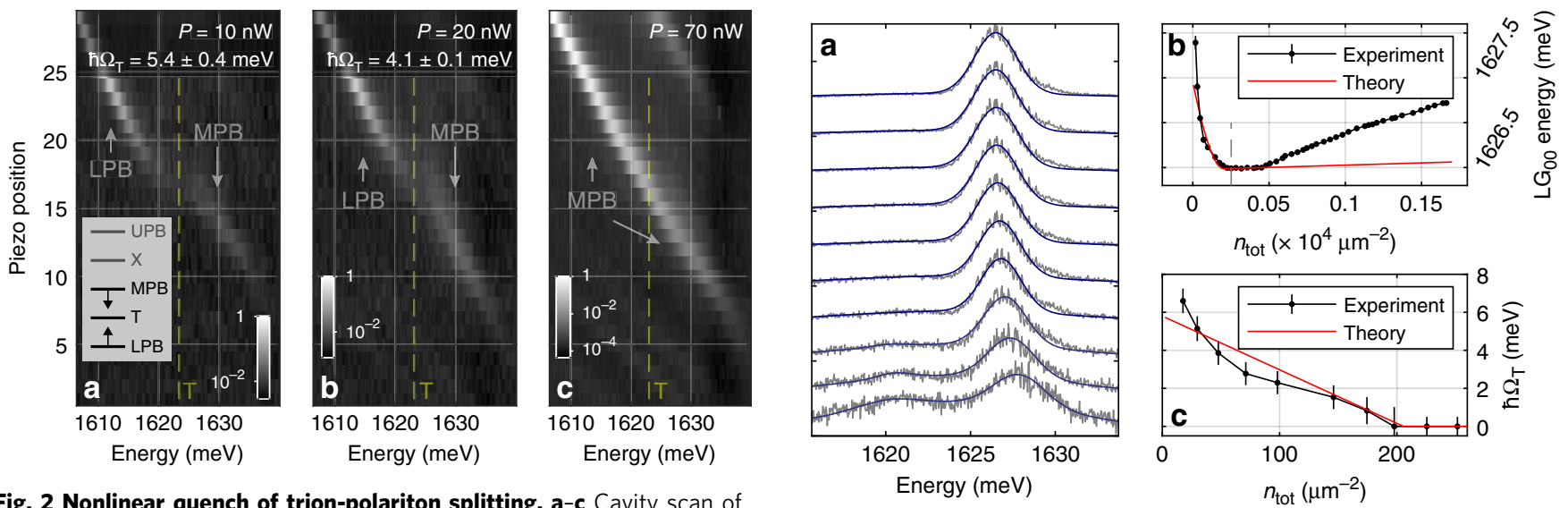

Fig. 2 Nonlinear quench of trion-polariton splitting. a-c Cavity scan of $L G_{00}$ mode across the trion resonance for different pump powers: 10, 20 and $70 \mathrm{nW}$, respectively. The peak energy of the pump laser is fixed at $\sim 1.62 \mathrm{eV}$. Pseudo-colour scale is logarithmic. Estimates for the trion Rabi splittings are obtained by fitting extracted peak positions for each piezo step with a coupled oscillator model. Inset: schematic diagram showing how the energies MPB and LPB are renormalised in the case of phase space filling effect leading to reduction of Rabi-splitting between trion level and the bare photon mode.

Supplementary Note 3.2). The effective strength of the trionpolariton nonlinearity $\beta_{\mathrm{T}}^{\text {eff }}$ responsible for the quenching of strong coupling (and large energy shifts) is defined as $\beta_{\mathrm{T}}^{\text {eff }}=-d\left(\hbar \Omega_{\mathrm{T}}\right) / d n_{\text {tot }}{ }^{28}$. From Fig. $3 \mathrm{c}$ we deduce an average value of $\beta_{\mathrm{T}}^{\text {eff }} \simeq 37 \pm 3 \mu \mathrm{eV} \cdot \mu \mathrm{m}^{2}$ (see Supplementary Note 1.4). Note, that the $3 \mu \mathrm{eV} \cdot \mu \mathrm{m}^{2}$ error above is a random error arising from the uncertainty in $\hbar \Omega_{\mathrm{T}}$ in Fig. $3 \mathrm{c}$ and does not account for possible systematic error of $n_{\text {tot }}$, which is discussed below and in Supplementary Information.

To explain the observed result we account for phase space filling effects which become important at increasing excited trion density (see Supplementary Note 3). This leads to the quenching of the collective trion oscillator strength: as more and more trions are excited in the system, the extra injected photons have less

Fig. 3 Trion-polariton nonlinearity. We consider large cavity-exciton detuning $\delta_{\mathrm{C}-\mathrm{X}}=-15.4 \mathrm{meV}$. a Spectra of the MPB and LPB for the excitation power from 5 to $50 \mathrm{nW}$ (power increases from bottom to top). The MPB and LPB peaks are fitted with Gaussians. The fitting of the exciton-like LPB peak (lower energy peak) is performed for the first 4 powers, since it becomes broad and weak at higher densities and the fitting procedure is not reliable. $\mathbf{b}$ Extracted peak positions of the MPB mode vs estimated total exciton-trion density. c Trion-polariton Rabi-splitting vs exciton-trion density. Red solid curves in $\mathbf{b}$, c correspond to the theoretical modelling results. The error bars $[95 \%$ confidence interval $(\mathrm{Cl})]$ are estimated taking into account the random error in determination of the peak positions in $\mathbf{a}$ as well as possible systematic error $\left(\Delta \Omega_{\mathrm{T}} \sim 0.5 \mathrm{meV}\right)$ due to the uncertainty of the fitting parameters in the coupled oscillators model.

electrons and trions to couple to and to form trion-polaritons. As a result the collapse of strong photon-trion coupling occurs at the density of excited trions equal to half the density of available free electrons, $n_{\mathrm{T}} \approx n_{\mathrm{e}} / 2$. The results of the theoretical modelling (see 'Methods') are shown by the red solid curves in Fig. 3b, c reproducing the experimental red shift of $E_{\mathrm{MPB}}$ and the corresponding reduction of $\hbar \Omega_{\mathrm{T}}$. Overall, the trion Rabisplitting can be approximated as $\Omega_{\mathrm{T}}\left(n_{\mathrm{T}}\right) \approx \Omega_{\mathrm{T}}(0)\left(1-2 n_{\mathrm{T}} / n_{\mathrm{e}}\right)$, 


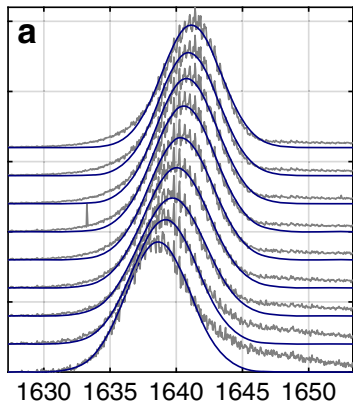

Energy (meV)

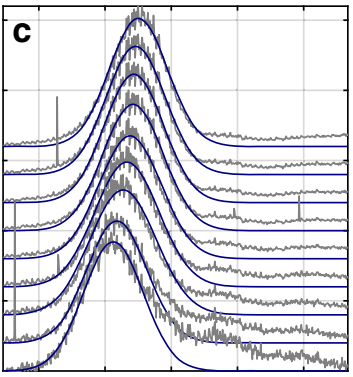

16301635164016451650 Energy (meV)
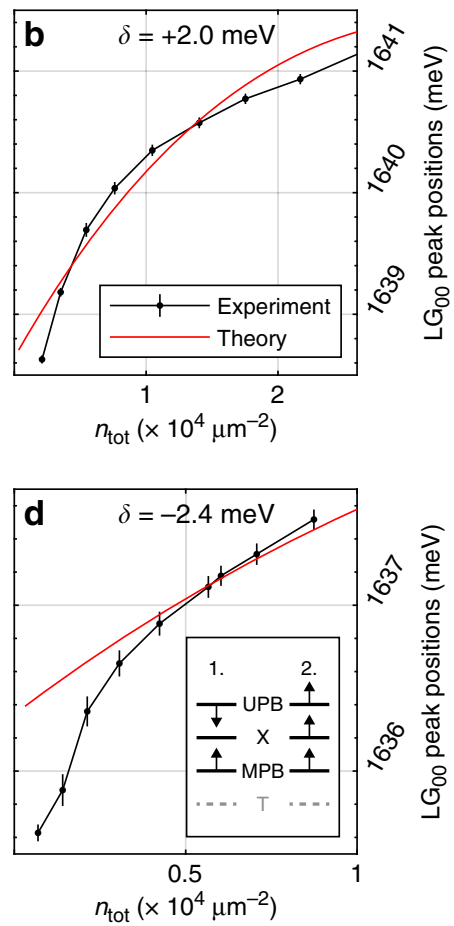

Fig. 4 Neutral exciton-polariton nonlinearity. a, b Small positive detuning $\delta_{\mathrm{C}-\mathrm{X}}=+2.0 \mathrm{meV}$ is considered. a Spectra of the MPB $L G_{00}$ mode for the pump powers from 1 to $9 \mu \mathrm{W}$ (power increases from bottom to top). b Extracted peak positions of the mode vs. exciton density. c, d Small negative detuning $\delta_{\mathrm{C}-\mathrm{X}}=-2.4 \mathrm{meV}$ is considered. c Spectra of the MPB $L G_{00}$ mode for the pump powers from 1 to $9 \mu \mathrm{W}$ (power increases from bottom to top). d Extracted peak positions of the mode vs. estimated exciton density. The error bars $(95 \% \mathrm{Cl})$ in $(\mathbf{b}-\mathbf{d})$ are deduced from the fitting procedure in $(\mathbf{a}-\mathbf{c})$. Red solid curves correspond to the theoretical modelling results. Inset: schematic diagrams showing how the MPB and UPB shift in the case of phase space filling (Mechanism 1 leading to reduction of Rabi-splitting between neutral exciton level and the bare photon mode) and the neutral exciton blueshift (Mechanism 2).

and the value of theoretical nonlinearity is given by $\beta_{\mathrm{T}}^{\text {eff }}=$ $2 \hbar \Omega_{\mathrm{T}}(0) / n_{\mathrm{e}} \approx 30 \mu \mathrm{eV} \cdot \mu \mathrm{m}^{2}$ for $n_{\mathrm{e}} \approx 400 \mu \mathrm{m}^{-2}$ and $\hbar \Omega_{\mathrm{T}}(0) \approx$ $5.8 \mathrm{meV}$ (see Supplementary Note 3.5) in agreement with the experimental value of $37 \mu \mathrm{eV} \cdot \mu \mathrm{m}^{2}$. It is the low electron density and high oscillator strength per single trion (large $\hbar \Omega_{\mathrm{T}}(0)$ ), that lead to the high value of trion-polariton nonlinearity.

Nonlinear neutral exciton-polaritons. Next we study the neutral exciton-polariton nonlinearity, which may arise from: (1) the reduction of exciton-photon Rabi-splitting $\hbar \Omega_{\mathrm{X}}$, and/or (2) blueshift of the neutral exciton level $E_{\mathrm{X}}{ }^{28}$. Both mechanisms should lead to blueshift of the MPB. The blueshift of the MPB peak associated with the neutral exciton-polariton nonlinearity is observed in Fig. $3 \mathrm{~b}$ for $\delta_{\mathrm{C}-\mathrm{X}}=-15.4 \mathrm{meV}$ at $n_{\text {tot }}>5 \times 10^{2} \mu \mathrm{m}^{-2}$ above the threshold of strong trion-photon coupling collapse. We further studied neutral exciton-polariton nonlinearity for several photon-exciton detunings in the range from $+8.8 \mathrm{meV}$ to -2.4 $\mathrm{meV}$, where the trion fraction is negligible ( $\sim 3 \%$ or less; see Table II in Supplementary Information). The central laser frequency was shifted to be in resonance with the MPB in each case. Substantial blueshifts of MPB mode of the order of $2 \mathrm{meV}$ are observed at much higher excitation power in the range from 1 to $9 \mu \mathrm{W}$ (see Fig. 4a, c). The MPB energy depends sublinearly on the total exciton/trion density $n_{\text {tot }}$ as shown in Fig. $4 \mathrm{~b}$, d. In this power range the density of excited neutral excitons $n_{\mathrm{X}}$ is much

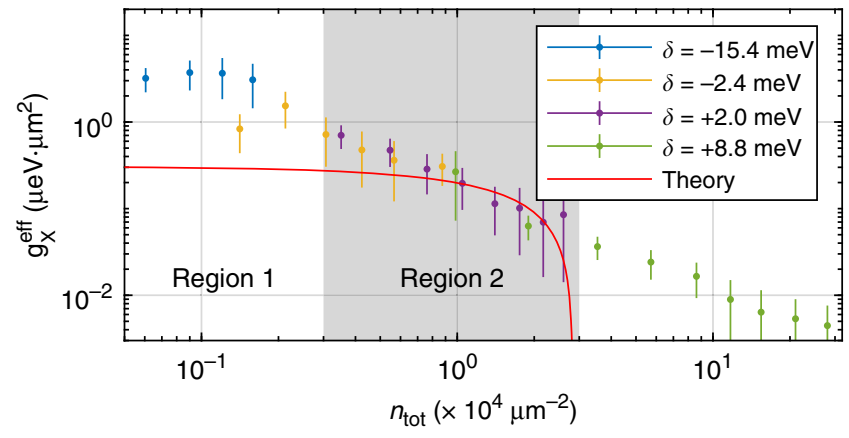

Fig. 5 Strength of nonlinearity vs pump. The experimental effective interaction constant $g_{\mathrm{X}}^{\text {eff }}$ is shown as a function of the estimated exciton density, $n_{\text {tot. }}$. The $g_{X}^{\text {eff }}$ is deduced from the energy blueshift of MPB (see Supplementary Note 1.5). The data correspond to four different cavity-exciton detunings $(\delta)$ : $+8.8 \mathrm{meV}$ (olive), $+2.0 \mathrm{meV}$ (purple), $-2.4 \mathrm{meV}$ (orange), and $-15.4 \mathrm{meV}$ (blue). The error bars $(95 \% \mathrm{Cl}$ ) are deduced taking into account errors in determining the MPB peak positions at each pump power (exciton density). The red solid curve corresponds to the theoretical values.

higher than that of trions and $n_{\text {tot }}$ is dominated mostly by neutral excitons $\left(n_{\mathrm{tot}} \gg n_{\mathrm{e}}, n_{\mathrm{tot}} \approx n_{\mathrm{X}}\right)$.

As we discuss in Supplementary Note 1.5 at intermediate densities the optical nonlinearity arises mainly from the exciton blueshift (Mechanism 2) ${ }^{28}$, which is characterised by the parameter $g_{\mathrm{X}}^{\text {eff }}=d E_{\mathrm{X}} / d n_{\mathrm{tot}}$. The $g_{\mathrm{X}}^{\text {eff }}$ is expected to be constant in a system where only pair exciton-exciton interactions are important $^{28}$. By contrast, Fig. 5 shows that the experimental $g_{\mathrm{X}}^{\text {eff }}$ decreases with $n_{\text {tot }}$ from $\simeq 2-5 \mu \mathrm{eV} \cdot \mu \mathrm{m}^{2}$ at $n_{\mathrm{to}} \sim 10^{3} \mu \mathrm{m}^{-2}$ to $\simeq 0.01 \mu \mathrm{eV} \cdot \mu \mathrm{m}^{2}$ at $n_{\text {tot }} \sim 10^{5} \mu \mathrm{m}^{-2}$, which suggests the importance of higher order exciton-exciton interactions. The lower values $\simeq 0.05 \mu \mathrm{eV} \cdot \mu \mathrm{m}^{2}$ are similar to the values reported in $\mathrm{WS}_{2}$ waveguide structures, where only very high excitation powers were used ${ }^{29}$.

To describe the observed neutral exciton-nonlinearity we developed a model taking into account the two- and three-exciton exchange processes 30,31 (see 'Methods' and Supplementary Note 2). At $3 \times 10^{3}<n_{\text {tot }}<3 \times 10^{4} \mu \mathrm{m}^{-2}$ within an experimental error there is agreement between theory and experiment (Fig. 5, region 2). The values of $g_{\mathrm{X}}^{\text {eff }}$ in exciton density region 2 are also in agreement with the values of the exciton-exciton interaction parameter in monolayer $\mathrm{WSe}_{2}$ characterising excitation induced exciton broadening in the same density range ${ }^{32}$. The theory also qualitatively reproduces the sublinear shifts in Fig. $4 \mathrm{~b}$ and d. At $n_{\text {tot }}>3 \times 10^{4} \mu \mathrm{m}^{-2}$ our theory is not applicable anymore, since in this case higher order nonlinearities should be taken into account. By contrast, at $n_{\text {tot }}<3 \times 10^{3} \mu \mathrm{m}^{-2}$ the model accounting only for exciton-exciton interactions results in the very weak theoretical blueshift of $E_{\mathrm{MPB}}$, much smaller than that observed in the experiment in Fig. 3b. This is also reflected in Fig. 5, where at $n_{\text {tot }}<3 \times 10^{3} \mu \mathrm{m}^{-2}($ region 1$)$ the theoretical $g_{\mathrm{X}}^{\text {th }} \sim 0.3 \mu \mathrm{eV} \cdot \mu \mathrm{m}^{2}$ is observed to be below the corresponding experimental values $g_{\mathrm{X}}^{\text {eff }} \sim 0.5-3 \mu \mathrm{eV} \cdot \mu \mathrm{m}^{2}$. Such a discrepancy indicates that at these exciton densities the trion-mediated exciton-exciton interactions, which are characterised by increased scattering cross-section and number of exchange processes, might play an important role in the observed large polariton blueshift (see Supplementary Note 3.6).

\section{Discussion}

In this work we measure the nonlinearity due to trion-polaritons with respect to that due to neutral exciton-polaritons. We observed 
that depending on the exciton-photon energy detuning the trionpolariton nonlinearity is $10-100$ times larger than that due to neutral exciton-polaritons. For example, the trion-polariton energy redshift of the order of $1 \mathrm{meV}$ is observed at excitation power of $\sim 20 \mathrm{nW}$ (see Fig. 3a) at $\delta_{\mathrm{C}-\mathrm{X}}=-15 \mathrm{meV}$, whereas in the case of neutral exciton-polaritons the same energy shift is observed at excitation powers of $\sim 400 \mathrm{nW}$ and $\sim 4 \mu \mathrm{W}$ at $\delta_{\mathrm{C}-\mathrm{x}}=-15 \mathrm{meV}$ and $\delta_{\mathrm{C}-\mathrm{X}}=2 \mathrm{meV}$ in Figs. $3 \mathrm{~b}$ and 4 , respectively. This is consistent with fact that the deduced average value of $\beta_{\mathrm{T}}^{\text {eff }} \simeq 37 \mu \mathrm{eV} \cdot \mu \mathrm{m}^{2}$ is $10-100$ times larger than the values $g_{\mathrm{X}}^{\text {eff }}$ observed in Fig. 5 at $n_{\text {tot }}<$ $10^{4} \mu \mathrm{m}^{-2}$.

Determination of the measured absolute values $\beta_{\mathrm{T}}^{\text {eff }}$ and $g_{\mathrm{X}}^{\text {eff }}$ requires careful deduction of the total polariton (exciton/trion) density excited with a single resonant pulse in the system in order to minimise possible systematic errors. The most accurate way to do this would be to use the incident energy of the excitation pulse and the coupling efficiency of the external radiation to the $0 \mathrm{D}$ polariton mode. In our experiment, this coupling efficiency is not known, since the excitation beam does not match well the spatial profile of the polariton mode. Instead, we deduce the total exciton/ trion density using the transmitted power and the ratio of the measured polariton linewidth to that of the bare cavity mode linewidth. The latter gives an upper bound on the ratio of the polaritons absorbed into the reservoir to polaritons emitted into free space since scattering into the reservoir cannot be faster than the total loss rate of polaritons (see discussion in Supplemental Material for more details). The accuracy of this method can be judged by the fact the values of $g_{\mathrm{X}}^{\text {eff }}$ of about $1-0.3 \mu \mathrm{eV} \mu \mathrm{m}^{2}$ at exciton densities in the range $10^{3}-10^{4} \mu \mathrm{m}^{-2}$ derived in the current manuscript are consistent within a factor of $2-3$ with $g_{\mathrm{X}}^{\text {eff }} \sim$ $1 \pm 0.4 \mu \mathrm{eV} \cdot \mu^{2}$ measured in ref. 33 for neutral excitonpolaritons realised in a slab waveguide photonic crystal in the same density range. In ref. ${ }^{33}$ the exciton density was derived accurately using femtosecond excitation and detection and measurement of the absolute power of the resonant excitation pulse and the coupling efficiency of the incident radiation to the polariton mode. In that case polariton absorption is not relevant. Since in the current work the trion-polariton nonlinearity is measured with respect to the neutral exciton-polariton nonlinearity we can take $g_{\mathrm{X}}^{\text {eff }}$ as a reference point and confirm that the value $\beta_{\mathrm{T}}^{\text {eff }} \simeq 37 \mu \mathrm{eV} \cdot \mu \mathrm{m}^{2}$ is likely to be accurate within a factor of two to three as well. Good agreement between our theory of the trion-polariton nonlinearity (which does not use fitting parameters) and the experiment (Fig. 3c) further supports this statement.

Notably, the deduced trion-polariton nonlinearity is of the same order as that observed in microcavities with a single quantum $\operatorname{dot}^{2,3}$, where strong renormalisation of Rabisplitting $(\sim 100 \mu \mathrm{eV})$ occurs at a single photon level ${ }^{2}$. The average value of $\beta_{\mathrm{T}}^{\text {eff }} \simeq 37 \mu \mathrm{eV} \cdot \mu \mathrm{m}^{2}$ is also $\sim 5-10$ times larger ${ }^{8,28}$ or comparable $e^{9,1}$ to the reported coefficients characterising neutral exciton-polariton nonlinearity in GaAs system. In terms of future perspectives the value of $\beta_{\mathrm{T}}^{\text {eff }}$ can be further verified by studying the second-order correlation function of the emission from the resonantly driven polariton mode ${ }^{13,14}$. Strong interactions can lead to polariton blockade and antibunching ${ }^{13}$. As we elaborate theoretically in Supplementary Note 4 polariton quantum effects can potentially be realised in high- $\mathrm{Q}$ microcavities with embedded high quality homogeneous TMDC samples ${ }^{34}$, where very narrow polariton resonances with a linewidth given just by the cavity mode lifetime could be achieved.

It is also useful to relate parameter $\beta_{\mathrm{T}}^{\text {eff }}$ to nonlinear refractive index coefficient $n_{2}$ widely used in the field of nonlinear optics to characterise Kerr-like nonlinearity of materials. We estimated the value of $n_{2}$ per single flake associated with strong trion-photon coupling to be around $\sim 10^{-10} \mathrm{~m}^{2} \cdot \mathrm{W}^{-1}$ (see Supplementary
Note 1.7). This value is 3-5 orders of magnitude greater than nonlinear refractive coefficient of 2D TMDC materials and graphene studied in the weak light-matter coupling regime (see Supplementary Note 1.7). The nonlinear refractive index $n_{2}$ due to trion polaritons of a hybrid monolayer-cavity system is also found to be 3-4 orders magnitude larger than $n_{2}$ coefficient of widely used bulk optical materials (e.g., Si, AlGaAs, etc.) [see Supplementary Note 1.7].

In conclusion, we observed strong Kerr-like nonlinearity associated with trion-polaritons in a TMDC system due to phase space filling of trion states. The ratio of trion- to neutral exciton-polariton interaction strength is found to be in the range from 10 to 100 . The variation of neutral-exciton-polariton nonlinearity with density is attributed to higher order exciton-exciton and trion-exciton interactions. Our work paves the way towards development of scalable active nanophotonic devices based on 2D materials (where the exciton level is the same across large areas in contrast to $0 \mathrm{D}$ quantum dots) utilising the polariton nonlinearity for control of light by light, potentially at quantum level ${ }^{35}$.

\section{Methods}

Experimental technique. In our experiment a $\mathrm{MoSe}_{2}$ monolayer is covered with a monolayer of hexagonal boron nitride $(\mathrm{hBN})$ to protect $\mathrm{MoSe}_{2}$ from contamination. The heterostructure of $\mathrm{hBN} / \mathrm{MoSe}_{2}$ was fabricated using standard mechanical exfoliation technique and dry transfer methods. To form a microcavity the hBN/ $\mathrm{MoSe}_{2}$ structure was positioned on top of a flat distributed Bragg reflector (DBR) (bottom mirror) consisting of 13 pairs $\mathrm{SiO}_{2} / \mathrm{Ta}_{2} \mathrm{O}_{5}$ quarter-wave layers. The top mirror is a 13-pair DBR deposited on a hemispherical surface, which is fabricated using focused ion beam milling. The two mirrors were then aligned and brought into a close proximity to each other (the distance between the mirrors is set to $\sim 1 \mu \mathrm{m}$ ) using piezo nano-positioners forming an "open-cavity" system (see Fig. $1 \mathrm{c}^{26}$ ) with the resulting formation of discrete microcavity Laguerre-Gauss (LG) photonic modes. The open cavity system was positioned into bath cryostat at temperature of $4 \mathrm{~K}$. For resonant excitation we employed $100 \mathrm{fs}$ laser pulses with repetition rate $1 \mathrm{kHz}$. These were obtained from the frequency-doubled output of an optical parametric amplifier (Light-Conversion TOPAS) pumped by the $800 \mathrm{~nm}$ pulses from a Ti:Sapphire regenerative amplifier system (Spectra Physics Spitfire seeded by Spectra-Physics Mai-Tai Ti:Sapphire Oscillator). The excitation beam was focused into the spot size of about $5-10 \mu \mathrm{m}$ on the bottom flat mirror, so that only a fraction of the incident photons couples to the highly confined $L G_{00}$ cavity mode.

Theory methods. To describe the observed polariton nonlinearity we begin with the conceptually more simple case of a neutral exciton-polariton, where the trion fraction is negligible (Fig. 4). Aiming to describe the influence of the exciton density $\left(n_{\mathrm{X}}\right)$ increase, we note that the Rabi frequency $\Omega_{\mathrm{X}}$ corresponds to the low intensity value $\Omega_{\mathrm{X}}(0)$ only for the case when excitons are ideal bosons described by a creation operator $\hat{X}_{\mathbf{k}}^{\dagger}$. The deviation of statistics coming from the Pauli principle leads to their changed commutation relations, $\left[\hat{X}_{\mathbf{k}}, \hat{X}_{\mathbf{k}^{\prime}}^{\dagger}\right]=\delta_{\mathbf{k}, \mathbf{k}^{\prime}}-D_{\mathbf{k}, \mathbf{k}^{\prime}}{ }^{36}$, where $D_{\mathbf{k}, \mathbf{k}^{\prime}}$ is a non-bosonicity parameter. In the analysis we consider density-dependent Rabi-splitting $\hbar \Omega_{\mathrm{X}}\left(n_{\mathrm{X}}\right)$ and nonlinear shift of the exciton level $E_{\mathrm{X}}\left(n_{\mathrm{X}}\right)$ (see Supplementary Note 2 for the details). The modified coupling reads

$$
\Omega_{\mathrm{X}}\left(n_{\mathrm{X}}\right)=\Omega_{\mathrm{X}}(0)\left(1-\frac{8 \pi}{7} n_{\mathrm{X}} a_{\mathrm{B}}^{2}+\frac{384 \pi^{2}}{455} n_{\mathrm{X}}^{2} a_{\mathrm{B}}^{4}\right),
$$

where $a_{\mathrm{B}}$ is an exciton Bohr radius in TMDC. The nonlinear shift of the exciton energy arising from the Coulomb scattering and deviation of exciton statistics from that of ideal bosons, reads

$$
\begin{aligned}
E_{\mathrm{X}}\left(n_{\mathrm{X}}\right)= & E_{\mathrm{X}}(0)+\frac{8}{\pi} \frac{e^{2}}{4 \pi \epsilon_{0} \kappa a_{\mathrm{B}}} \mathcal{I}_{4}\left(r_{0}\right) n_{\mathrm{X}} a_{\mathrm{B}}^{2} \\
& -\frac{128}{5} \frac{e^{2}}{4 \pi \epsilon_{0} \kappa a_{\mathrm{B}}}\left[5 \mathcal{I}_{6}\left(r_{0}\right)-2 \mathcal{I}_{4}\left(r_{0}\right)\right] n_{\mathrm{X}}^{2} a_{\mathrm{B}}^{4},
\end{aligned}
$$

where $e$ is an electron charge, $\epsilon_{0}$ is vacuum permittivity, $\kappa$ denotes average dielectric permittivity, $r_{0}$ is a screening parameter, and $\mathcal{I}_{4,6}\left(r_{0}\right)$ are dimensionless integrals. The linear $\left(\propto n_{\mathrm{X}}\right)$ and quadratic terms $\left(\propto n_{\mathrm{X}}^{2}\right)$ in Equations (1) and (2) take into account the two- and three-exciton exchange processes ${ }^{30,31}$, respectively.

We apply the above theory to explain the nonlinear MPB blueshift when trion contribution can be omitted. To do so, the exciton-photon Hamiltonian is diagonalised accounting both for $E_{\mathrm{X}}\left(n_{\mathrm{X}}\right)$ and $\Omega_{\mathrm{X}}\left(n_{\mathrm{X}}\right)$, and energy for the lower polariton branch is considered ${ }^{28}$. The theoretical results are shown in Fig. $4 \mathrm{~b}, \mathrm{~d}$ by the red solid curves. Performing the variational procedure to calculate the 
properties of excitons and matching the binding energy to experimental value, we set the exciton Bohr radius to $a_{\mathrm{B}}=0.85 \mathrm{~nm}^{37}$ and qualitatively describe the sublinear dependence of polariton blueshift (see Supplementary Note 2). By differentiating Eqs. (1) and (2) over $n_{\mathrm{X}}$ we can obtain the corresponding theoretical $\beta_{\mathrm{X}}^{\text {th }}$ and $g_{\mathrm{X}}^{\text {th }}$ parameters, which characterize the neutral exciton-polariton nonlinearity due to reduction of exciton-polariton Rabi-splitting and the exciton level blueshift, respectively (see Supplementary Note 1.5).

Gaining the knowledge from the exciton case, we consider the trion-dominated regime (Figs. 2 and 3). The excitation process then corresponds to the creation of a trion from a free electron ${ }^{24,38,39}$. We account for phase space filling effects which become important at increasing excited trion density (see Supplementary Note 3). This leads to the quenching of the collective trion oscillator strength. Taking into account the deviation of statistics for trions from that of ideal fermions ${ }^{40}$ we calculate the influence of phase space filling on the trion Rabi frequency $\Omega_{\mathrm{T}}\left(n_{\mathrm{T}}, \lambda_{1,2}\right)$ as a function of density and variational parameters for the trion wavefunction $\lambda_{1,2}$. To describe Fig. $3 \mathrm{~b}, \mathrm{c}$ we perform the diagonalisation of the full photon-trion-exciton system with nonlinear contributions to both trion and exciton modes. We use $\lambda_{1}=0.86 \mathrm{~nm}, \lambda_{2}=2.54 \mathrm{~nm}$, obtained by the variational procedure, in line with computational trion studies in $\mathrm{MoSe}_{2}{ }_{2}^{41}$. The results are shown by the red solid curves in Fig. $3 \mathrm{~b}, \mathrm{c}$ reproducing the experimental red shift of $E_{\mathrm{MPB}}$ and reduction of $\Omega_{\mathrm{T}}$.

\section{Data availability}

Data supporting this study are openly available from the University of Sheffield repository: https://doi.org/10.15131/shef.data.12523217.

\section{Code availability}

All the detailed theoretical derivations are presented in the Supplementary materials and can be easily implemented in, for example, Mathematica package.

Received: 11 May 2020; Accepted: 24 June 2020;

Published online: 17 July 2020

\section{References}

1. Chang, D. E., Vuletić, V. \& Lukin, M. D. Quantum nonlinear optics-photon by photon. Nat. Photonics 8, 685-694 (2014).

2. Volz, T. et al. Ultrafast all-optical switching by single photons. Nat. Photonics 6, 605-609 (2012).

3. Sun, S., Kim, H., Luo, Z., Solomon, G. S. \& Waks, E. A single-photon switch and transistor enabled by a solid-state quantum memory. Science 361, 57-60 (2018).

4. Maser, A., Gmeiner, B., Utikal, T., Götzinger, S. \& Sandoghdar, V. Few-photon coherent nonlinear optics with a single molecule. Nat. Photonics 10, 450-453 (2016).

5. Volz, J., Scheucher, M., Junge, C. \& Rauschenbeutel, A. Nonlinear $\pi$ phase shift for single fibre-guided photons interacting with a single resonatorenhanced atom. Nat. Photonics 8, 965-970 (2014).

6. Universal Themes of Bose-Einstein Condensation (eds Proukakis, N. P., Snoke, D. W. \& Littlewood, P. B.) https://doi.org/10.1017/97813316084366 (Cambridge University Press, 2017).

7. Sanvitto, D. \& Kéna-Cohen, S. The road towards polaritonic devices. Nat. Mater. 15, 1061 (2016).

8. Walker, P. M. et al. Ultra-low-power hybrid light matter solitons. Nat. Commun. 6, 8317 (2015)

9. Ferrier, L. et al. Interactions in confined polariton condensates. Phys. Rev. Lett. 106, 126401 (2011).

10. Rodriguez, S. R. K. et al. Interaction-induced hopping phase in drivendissipative coupled photonic microcavities. Nat. Commun. 7, 11887 (2016).

11. Cuevas, Á. et al. First observation of the quantized exciton-polariton field and effect of interactions on a single polariton. Sci. Adv. 4, eaao6814 (2018).

12. Sich, M., Skryabin, D. V. \& Krizhanovskii, D. N. Soliton physics with semiconductor exciton-polaritons in confined systems. Comptes Rendus Phys. 17, 908-919 (2016).

13. Muñoz-Matutano, G. et al. Emergence of quantum correlations from interacting fibre-cavity polaritons. Nat. Mater. 18, 213-218 (2019).

14. Delteil, A. et al. Towards polariton blockade of confined exciton-polaritons Nat. Mater. 18, 219-222 (2019).

15. Ballarini, D. et al. All-optical polariton transistor. Nat. Commun. 4, 1778 (2013).

16. Sturm, C. et al. All-optical phase modulation in a cavity-polariton MachZehnder interferometer. Nat. Commun. 5, 3278 (2014).

17. Wang, G. et al. Colloquium: Excitons in atomically thin transition metal dichalcogenides. Rev. Mod. Phys. 90, 021001 (2018).

18. Schneider, C., Glazov, M. M., Korn, T., Höfling, S. \& Urbaszek, B. Twodimensional semiconductors in the regime of strong light-matter coupling. Nat. Commun. 9, 2695 (2018).
19. Gu, T. et al. Regenerative oscillation and four-wave mixing in graphene optoelectronics. Nat. Photonics 6, 554-559 (2012).

20. Mak, K. F., Lee, C., Hone, J., Shan, J. \& Heinz, T. F. Atomically thin $\mathrm{MoS}_{2}$ : a new direct-gap semiconductor. Phys. Rev. Lett. 105, 136805 (2010).

21. Dufferwiel, S. et al. Exciton-polaritons in van der Waals heterostructures embedded in tunable microcavities. Nat. Commun. 6, 8579 (2015).

22. Liu, X. et al. Strong light-matter coupling in two-dimensional atomic crystals. Nat. Photonics 9, 30-34 (2014).

23. Zhang, L., Gogna, R., Burg, W., Tutuc, E. \& Deng, H. Photonic-crystal exciton-polaritons in monolayer semiconductors. Nat. Commun. 9, 713 (2018).

24. Rapaport, R. et al. Negatively charged quantum well polaritons in a GaAs/ AlAs microcavity: An analog of atoms in a cavity. Phys. Rev. Lett. 84, $1607-1610$ (2000).

25. Sidler, M. et al. Fermi polaron-polaritons in charge-tunable atomically thin semiconductors. Nat. Phys. 13, 255-261 (2017).

26. Giriunas, L. et al. Formation of a macroscopically occupied polariton state in a tunable open-access microcavity under resonant excitation. J. Appl. Phys. 124, 025703 (2018).

27. Walker, P. M. et al. Dark solitons in high velocity waveguide polariton fluids. Phys. Rev. Lett. 119, 097403 (2017)

28. Brichkin, A. S. et al. Effect of Coulomb interaction on exciton-polariton condensates in GaAs pillar microcavities. Phys. Rev. B 84, 195301 (2011).

29. Barachati, F. et al. Interacting polariton fluids in a monolayer of tungsten disulfide. Nat. Nanotech. 13, 906-909 (2018).

30. Tassone, F. \& Yamamoto, Y. Exciton-exciton scattering dynamics in a semiconductor microcavity and stimulated scattering into polaritons. Phys. Rev. B 59, 10830-10842 (1999).

31. Shahnazaryan, V., Iorsh, I., Shelykh, I. A. \& Kyriienko, O. Exciton-exciton interaction in transition-metal dichalcogenide monolayers. Phys. Rev. B 96, 115409 (2017).

32. Moody, G. et al. Intrinsic homogeneous linewidth and broadening mechanisms of excitons in monolayer transition metal dichalcogenides. Nat. Commun. 6, 8315 (2015).

33. Kravtsov, V. et al. Nonlinear polaritons in a monolayer semiconductor coupled to optical bound states in the continuum. Light Sci. Appl. 9, 56 (2020).

34. Cadiz, F. et al. Excitonic Linewidth Approaching the Homogeneous Limit in $\mathrm{MoS}_{2}$-Based van der Waals Heterostructures. Phys. Rev. X 7, 021026 (2017).

35. Kyriienko, O., Krizhanovskii, D.N. \& Shelykh, I.A. Nonlinear quantum optics with trion-polaritons in $2 \mathrm{~d}$ monolayers: conventional and unconventional photon blockade.Preprint at https://arxiv.org/abs/1910.11294 (2019).

36. Combescot, M., Betbeder-Matibet, O. \& Dubin, F. The many-body physics of composite bosons. Phys. Rep. 463, 215-320 (2008).

37. Kylänpää, I. \& Komsa, H.-P. Binding energies of exciton complexes in transition metal dichalcogenide monolayers and effect of dielectric environment. Phys. Rev. B 92, 205418 (2015).

38. Rapaport, R., Cohen, E., Ron, A., Linder, E. \& Pfeiffer, L. N. Negatively charged polaritons in a semiconductor microcavity. Phys. Rev. B 63, 235310 (2001).

39. Shiau, S.-Y., Combescot, M. \& Chang, Y.-C. Trion ground state, excited states, and absorption spectrum using electron-exciton basis. Phys. Rev. B 86, 115210 (2012).

40. Combescot, M. \& Betbeder-Matibet, O. General many-body formalism for composite quantum particles. Phys. Rev. Lett. 104, 206404 (2010).

41. Kidd, D. W., Zhang, D. K. \& Varga, K. Binding energies and structures of twodimensional excitonic complexes in transition metal dichalcogenides. Phys. Rev. B 93, 125423 (2016).

\section{Acknowledgements}

The authors acknowledge the support from the UK EPSRC grants EP/N031776/1, EP/ M012727/1, EP/P026850/1 and from the mega-grant No. 14.Y26.31.0015 of the Ministry of Education and Science of the Russian Federation. O.K. thanks to ITMO Fellowship and Professorship Program. V.S. and I.A.S. acknowledge support from goszadanie no 3.2614.2017/4.6 of the Ministry of Education and Science of the Russian Federation and Icelandic research fund, grant No. 163082-051. A.C. and A.I.T. thank the financial support of the European Union's Horizon 2020 research and innovation programme under ITN Spin-NANO Marie Sklodowska-Curie grant agreement no. 676108.

\section{Author contributions}

R.P.A.E., M.S. and F.B.A. performed the experiments and analysed the data; F.W. and A.C. provided the samples; P.M.W. designed the cavity structure; O.K., V.S. and I.A.S. provided the theoretical explanation; M.S., O.K., P.M.W., M.S.S., A.I.T., F.B.A. and D.N.K. wrote the paper; D.N.K. conceived the project and oversaw the experiment.

\section{Competing interests}

The authors declare no competing interests. 


\section{Additional information}

Supplementary information is available for this paper at https://doi.org/10.1038/s41467$020-17340-z$

Correspondence and requests for materials should be addressed to O.K. or D.N.K.

Peer review information Nature Communications thanks Nathaniel P. Stern and the other, anonymous reviewer(s) for their contribution to the peer review of this work.

Reprints and permission information is available at http://www.nature.com/reprints

Publisher's note Springer Nature remains neutral with regard to jurisdictional claims in published maps and institutional affiliations. (c) (i) Open Access This article is licensed under a Creative Commons Attribution 4.0 International License, which permits use, sharing, adaptation, distribution and reproduction in any medium or format, as long as you give appropriate credit to the original author(s) and the source, provide a link to the Creative Commons license, and indicate if changes were made. The images or other third party material in this article are included in the article's Creative Commons license, unless indicated otherwise in a credit line to the material. If material is not included in the article's Creative Commons license and your intended use is not permitted by statutory regulation or exceeds the permitted use, you will need to obtain permission directly from the copyright holder. To view a copy of this license, visit http://creativecommons.org/licenses/by/4.0/.

(C) The Author(s) 2020 